\title{
Produtos fitossanitários alternativos no controle da mosca-branca (Bemisia tuberculata) (Matile-Ferrero), na mandioca
}

\section{Alternative phytosanitary products to control of whitefly (Bemisia tuberculata) (Matile-Ferrero), in cassava}

\author{
Ana Raquel Rheinheimer ${ }^{1 *}$; Luis Francisco Angeli Alves²; Vanda Pietrowski; \\ Patrícia Paula Bellon ${ }^{4}$; Aline Monsani Miranda ${ }^{5}$ Diego Gazola ${ }^{6}$
}

\section{Resumo}

Este estudo teve como objetivo avaliar a eficiência de produtos fitossanitários alternativos sobre a oviposição e mortalidade de ninfas e adultos da mosca-branca (Bemisia tuberculata). Foi avaliada a ação inseticida dos produtos comerciais Planta Clean ${ }^{\circledR}$, Pironin ${ }^{\circledR}$, Mattan Plus ${ }^{\circledR}$ e Calda fertilizante foliar $^{\circledR}$, sobre ninfas de terceiro ínstar, por meio da pulverização direta dos produtos, sendo observada a mortalidade durante 10 dias. Para avaliar os efeitos desses produtos na deterrência de oviposição e mortalidade de adultos, as quatro folhas apicais de 25 plantas receberam aplicação dos produtos e foram posteriormente infestadas com 30 adultos de $B$. tuberculata. Os insetos foram mantidos em gaiolas adaptadas às folhas da planta e a avaliação do número de adultos mortos e o número de ovos nas folhas foi realizada $48 \mathrm{~h}$ após o início do experimento. Planta Clean ${ }^{\circledR}$ destacou-se no controle de ninfas $(54 \%$ de mortalidade) e para adultos, o melhor produto foi Pironin ${ }^{\circledR}(86,8 \%)$. Todos os produtos causaram deterrência de oviposição.

Palavras-chave: Manihot esculenta, controle alternativo, oviposição, inseticida

\begin{abstract}
This study aimed to evaluate the efficiency of alternative phytossanitary products on the oviposition and mortality of nymphs and adults of whitefly (Bemisia tuberculata). It was evaluated the insecticidal action of commercial products Plant Clean ${ }^{\circledR}$, Pironin ${ }^{\circledR}$, Mattan Plus ${ }^{\circledR}$ and Calda fertilizante foliar ${ }^{\circledR}$ on third instar nymphs, by products direct spraying, being the mortality observed during 10 days. To evaluate the effects of these products in oviposition deterrence and adult mortality, the four upper leaves of 25 plants were treated with products and then were infested with 30 adults of $B$. tuberculata. The insects were kept in cages adapted to plant leaves and evaluation of the number of dead adults and eggs on the leaves was done $48 \mathrm{~h}$ after the beginning of the study. Plant Clean ${ }^{\circledR}$ stood out on nymphs control (54\% mortality) and for adults, the best product was Pironin ${ }^{\circledR}(86.8 \%)$. All products caused deterrence in oviposition.
\end{abstract}

Key words: Manihot esculenta, alternative control, oviposition, insecticide

\footnotetext{
${ }^{1}$ Doutoranda do programa de Pós-Graduação em Agronomia pela Universidade Estadual do Oeste do Paraná, UNIOESTE, Marechal Cândido Rondon, PR. E-mail: anaraquel_bio@hotmail.com

${ }^{2}$ Prof. Associado do Centro de Ciências Biológicas e da Saúde, UNIOESTE, Cascavel, PR. Bolsista de Produtividade em Pesquisa/ CNPq. E-mail: luis.alves@unioeste.br

${ }^{3}$ Prof $f^{a}$ Adjunta do Centro de Ciências Agrárias, UNIOESTE, Marechal Cândido Rondon, PR. E-mail: vandapietrowski@gmail. com

${ }^{4}$ Doutoranda do Programa de Pós graduação em Entomologia e Conservação da Biodiversidade. Faculdade de Ciências Biológicas e Ambientais, FCBA, Universidade Federal da Grande Dourados, UFGD. Dourados, MS. E-mail: phatriciabellon@yahoo.com.br

${ }^{5}$ Acadêmica do Curso de Agronomia, Universidade Estadual do Oeste do Paraná, UNIOESTE. Marechal Cândido Rondon, PR. E-mail: liny_smi@hotmail.com

${ }^{6}$ Discente do Curso de Agronomia, Universidade Estadual do Oeste do Paraná, UNIOESTE. Marechal Cândido Rondon, PR. E-mail: gazolad@hotmail.com

* Autor para correspondência
} 
As moscas-brancas constituem um grave problema na produção de mandioca na região neotropical (BELLOTTI; SMITH; LAPOINTE, 1999). Os danos são causados por ninfas e adultos, sendo caracterizados de maneira direta pela sucção da seiva (provocando clorose, encarquilhamento de ponteiros e queda foliar) e, indiretamente, favorecendo o desenvolvimento da fumagina e pela transmissão de viroses (BELLOTTI et al., 2002).

No Brasil, nas áreas de cultivo de mandioca nos estados do Nordeste predomina a espécie Aleurothrixus aepim, enquanto que nos estados de Mato Grosso do Sul, Bahia e Rio de Janeiro ocorrem A. aepim, Bemisia tuberculata e Tetraleurodes variabilis (OLIVEIRA et al., 2001). No estado do Paraná, a espécie que ocorre nas lavouras do oeste do Estado foi identificada como $B$. tuberculata (RHEINHEIMER et al., 2009).

Poucas informações são disponíveis em relação ao controle das moscas-brancas nas diferentes culturas, que é baseado no uso exclusivo de inseticidas químicos. No entanto, segundo Moreira et al. (2006), tal prática não é recomendada para a cultura da mandioca, principalmente porque os inseticidas químicos convencionais, além de ineficientes não são registrados para a cultura (AGROFIT, 2011). Com isso, existe uma demanda crescente por métodos alternativos, visando ao manejo integrado para minimizar os danos, promover a redução populacional e reduzir os prejuízos advindos do seu ataque (MOREIRA et al., 2006).

Neste contexto, inserem-se produtos fitossanitários alternativos (PFA), com destaque para as plantas inseticidas, principalmente produtos à base de nim (Azadirachta indica), que atuam na repelência e mortalidade contra diversos insetos (FOSTER; HARRIS, 1997; MARTINEZ, 2002), além do piretro, encontrado naturalmente em flores de crisântemo (TAMM et al., 2004). Também têm sido comercializadas como PFA, para o controle de pragas na produção orgânica, as caldas fitoprotetoras e os biofertilizantes. A calda sulfocálcica, obtida por meio do tratamento térmico de enxofre e cal, tem sido citada como eficaz para controlar diversas pragas (MORANDI FILHO et al., 2006; VENZON et al., 2006).

Entretanto, estudos sobre os efeitos de PFA sobre mosca-branca na cultura da mandioca são escassos e a maioria das práticas atualmente utilizadas pelos produtores no controle desse inseto não tem sido eficiente. Neste sentido, a presente pesquisa foi conduzida com o objetivo de avaliar a eficiência de diferentes PFA sobre a oviposição e mortalidade de ninfas e adultos de $B$. tuberculata.

Os tratamentos avaliados e seus componentes e concentrações utilizadas estão descritos na Tabela 1. Para a testemunha foi utilizada apenas água destilada.

Tabela 1. Produtos fitossanitários alternativos, composições e concentrações utilizadas nos ensaios com ninfas e adultos de Bemisia tuberculata (Hemiptera: Aleyrodidae).

\begin{tabular}{lll}
\hline Produto comercial & Concentração utilizada & Componentes \\
\hline Planta Clean $^{\circledR}$ & $25{\mathrm{~mL} 1 \mathrm{~L}^{-1}}^{-1}$ & Extratos vegetais, ácidos graxos e sais minerais \\
Calda fertilizante foliar & $20 \mathrm{~mL} \mathrm{1L}$ & Mistura de enxofre e cal virgem \\
Pironim $^{\circledR}$ & $20 \mathrm{~mL} \mathrm{1L}^{-1}$ & $\begin{array}{l}\text { Nim }- \text { folhas, tortas de sementes e óleo; piretro } \\
\text { natural e extrato pirolenhoso }\end{array}$ \\
Mattam plus $^{\circledR}$ & $20 \mathrm{~mL} \mathrm{1L}^{-1}$ & Enxofre e nitrogênio \\
\hline
\end{tabular}

Fonte: Elaboração dos autores. 
Foram utilizadas plantas de mandioca da variedade Fécula Branca obtidas plantando-se manivas (cerca de $30 \mathrm{~cm}$ ), na posição vertical, em vasos com capacidade de $4 \mathrm{~L}$, utilizando-se solo Latossolo Vermelho $+10 \%$ de composto orgânico (palhada de braquiária, esterco de gado e esterco de galinha). As plantas foram mantidas em casa de vegetação e irrigadas diariamente até apresentarem oito folhas completamente desenvolvidas quando foram iniciados os testes, conforme metodologia descrita por Rheinheimer et al. (2009).

Os indivíduos de $B$. tuberculata foram coletados em plantas de mandioca cultivar Fécula Branca, utilizando-se aspiradores entomológicos confeccionados com tubos de borracha e tela antiafídica (30 cm de comprimento) e transferidos para as plantas para a realização dos ensaios.

Ação sobre ninfas de $B$. tuberculata. Para realização do ensaio, 25 plantas de mandioca foram transferidas para sala semi-climatizada (25 $\pm 5^{\circ} \mathrm{C}$ e fotoperíodo de $12 \mathrm{~h}$ ). As quatro folhas apicais (superiores) completamente desenvolvidas das plantas foram infestadas com 30 adultos não sexados de $B$. tuberculata por folha. Os insetos foram mantidos nas folhas com auxílio de gaiolas adaptadas às folhas da planta $(24 \times 30 \mathrm{~cm}-$ comprimento $\times$ largura), confeccionadas com tela antiafídica e plástico, segundo metodologia descrita por Bellon et al. (2011). Permitiu-se a oviposição por $48 \mathrm{~h}$, após a qual os adultos foram retirados e as plantas examinadas com auxílio de microscópio estereoscópico, mantendo-se cinco ovos/folha, eliminando-se o restante de ovos utilizando pincel fino. Quando as ninfas atingiram o terceiro ínstar (aproximadamente 18 dias) realizouse a aplicação dos produtos na face abaxial das folhas, sobre as ninfas, até o ponto de escorrimento (aproximadamente $1 \mathrm{~mL}$ folha ${ }^{-1}$ ), utilizando um aparelho aerógrafo acoplado a um compressor de ar (pressão de 7 lb). As ninfas foram observadas diariamente e a mortalidade anotada pelo período de 10 dias.
Ação sobre adultos e oviposição de $B$. tuberculata. Foram adotados os mesmos procedimentos descritos no ensaio anterior, exceto que a aplicação dos PFA foi realizada antes da infestação das plantas. Após a aplicação e secagem a sombra das folhas (aproximadamente 30 minutos), os adultos foram transferidos.

A avaliação foi realizada $48 \mathrm{~h}$ após a manutenção dos adultos nas folhas, contando-se, com auxílio de microscópio estereocópico, o número de adultos mortos e, o número de ovos presentes nas folhas. Calculou-se o efeito deterrente dos produtos à oviposição utilizando-se a fórmula

$$
P D=\frac{(N C-N T)}{(N C+N T)} \times 100,
$$

adaptada de Obeng-Ofori (1995), sendo: $\mathrm{PD}=\mathrm{a}$ porcentagem média de deterrência; $\mathrm{NC}=$ o número de ovos na testemunha; e NT = o número de ovos em cada tratamento com os produtos. Foi atribuída a seguinte classificação: Deterrente PD $>0$ e Neutro: $\mathrm{PD}<0$.

Análise estatística. Odelineamento experimental utilizado foi o Inteiramente Casualizado, com cinco tratamentos e 20 repetições, sendo cada folha considerada uma repetição. Os dados referentes à mortalidade total foram corrigidos pela fórmula de Schneider-Orelli (ALVES et al., 2005) e a eficiência dos produtos foi calculada pela fórmula de Abbott (ALVES et al., 2005). Aplicou-se o teste de homocedasticidade e detectou-se a necessidade de transformação dos dados em $\sqrt{x+0,5}$ sendo posteriormente submetidos à análise de variância (teste F) com médias as comparadas pelo teste de Tukey ( $\mathrm{P} \leq 0,05)$, utilizando-se o programa estatístico Sisvar (FERREIRA, 1992).

Ação sobre ninfas de $B$. tuberculata. O produto comercial Planta Clean ${ }^{\circledR}$ apresentou a maior ação inseticida para ninfas, causando mortalidade de $54 \%$, diferindo estatisticamente dos demais tratamentos. A Calda fertilizante foliar ${ }^{\circledR}$ e Pironin $^{\circledR}$ apresentaram mortalidade variando de 23,3 e $20,0 \%$, diferindo significativamente da testemunha (Tabela 2). 
Tabela 2. Mortalidade (\%) de ninfas e adultos de Bemisia tuberculata e eficiência (\%) dos tratamentos após aplicação dos PFA.

\begin{tabular}{lcccc}
\hline \multirow{2}{*}{ Tratamento } & \multicolumn{2}{c}{ Mortalidade (\%) } & \multicolumn{2}{c}{ Eficiência (\%) $^{\mathbf{2}}$} \\
\cline { 2 - 4 } & Ninfas & Adultos & Ninfas & Adultos \\
\hline Planta Clean $^{\circledR}$ & $54,0 \pm 1,32 \mathrm{a}$ & $17,4 \pm 1,32 \mathrm{~b}$ & $55,0 \pm 7,24$ & $11,5 \pm 1,18$ \\
Calda fertilizante foliar $^{\circledR}$ & $23,3 \pm 2,99 \mathrm{~b}$ & $68,5 \pm 6,01 \mathrm{a}$ & $24,0 \pm 3,11$ & $69,1 \pm 6,09$ \\
Pironin $^{\circledR}$ & $20,0 \pm 4,33 \mathrm{~b}$ & $86,8 \pm 3,43 \mathrm{a}$ & $21,0 \pm 4,53$ & $76,3 \pm 2,90$ \\
Mattan Plus $^{\circledR}$ & $4,0 \pm 2,75 \mathrm{c}$ & $12,8 \pm 2,66 \mathrm{~b}$ & $5,0 \pm 2,86$ & $4,8 \pm 0,82$ \\
Testemunha & $0,0 \pm 0,00 \mathrm{c}$ & $0,0 \pm 0,00 \mathrm{c}$ & - & - \\
\hline $\mathrm{CV}(\%)$ & 54,0 & 20,3 & \\
\hline DMS & 1,6 & 1,2 & \\
\hline &
\end{tabular}

Embora informações sobre a mortalidade de ninfas de $B$. tuberculata por PFA sejam escassas, há relatos do efeito causado por sementes de nim ( $A$. indica), sobre ninfas de $B$. tabaci com eficiência de 40\% (DREYER, 1990). Da mesma forma, Azevedo et al. (2005), comparando produtos comerciais sobre ninfas de B. tabaci biótipo $\mathrm{B}$, em condição de casa de vegetação obtiveram mortalidade de $66,5 \%$, para o óleo de nim e $67,4 \%$ para o extrato pirolenhoso. Também, mortalidade de $88,7 \%$ sobre ninfas de terceiro instar (BALDIN et al., 2007) e $97 \%$ de eficiência quando extrato aquoso de semente de nim foi aplicado sobre ninfas de primeiro ínstar (BLEICHER; GONÇALVES; SILVA, 2007). Estes resultados podem ser explicados devido à variação na quantidade de ingrediente ativo nas sementes de nim (BLEICHER; GONÇALVES; SILVA, 2007) que é o maior reservatório de azadiractina (BUTTERWORTH; MORGAN, 1971; JACOBSON, 1989; ROJATKAR et al., 1989).

No Brasil, alguns dos produtos à base de nim são comercializados sem registro oficial que informe a origem do produto e, como não existe uma garantia quanto à padronização da concentração do ingrediente ativo, tal fato pode resultar em diferenças na eficácia das formulações comerciais.
Nesse sentido, Martinez (2002) verificou variação de 2,90 a 6,10 $\mathrm{mg}$ de azadiractina $\mathrm{g}^{-1}$ de semente, em amostras coletadas em países das Américas, África e Ásia, sendo encontrada neste último continente a maior concentração, devido a ser o local de origem da planta. Além disso, a estrutura coletada da planta e sua idade, forma de acondicionamento do material, método de extração e condições climáticas são fatores que influenciam a quantidade de compostos presentes na semente (SCHMUTTERER, 1990; MARTINEZ; LIMA; BOIÇA JUNIOR, 1998).

Ação sobre adultos e oviposição de $B$. tuberculata. Os produtos $\operatorname{Pironin}^{\circledR}, \quad$ Calda fertilizante foliar ${ }^{\circledR}$, Planta Clean ${ }^{\circledR}$ e Mattan Plus ${ }^{\circledR}$ afetaram significativamente a sobrevivência dos adultos da mosca-branca. A mortalidade variou de 12,8 a $86,8 \%$ para esses produtos, destacando-se Pironin $^{\circledR}$ e Calda fertilizante foliar ${ }^{\circledR}$, com respectivo percentual de eficiência de 76,3 e 69,1 (Tabela 2). O produto Pironin ${ }^{\circledR}$ apresenta entre os componentes, o óleo de nim e o extrato pirolenhoso, sendo que o efeito inseticida de formulações à base destes compostos sobre adultos de $B$. tabaci biótipo B foi constatado por Azevedo et al. (2005), em condições de campo, em cultivo de melão. 
Tabela 3. Número médio de ovos por folha e deterrência na oviposição de Bemisia tuberculata em folhas tratadas com produtos fitossanitários alternativos.

\begin{tabular}{lcc}
\hline Tratamento & Número médio de ovos & Porcentagem de deterrência (PD) \\
\hline Calda fertilizante foliar $^{\circledR}$ & $3,3 \pm 0,58 \mathrm{a}^{2}$ & 90,8 \\
Pironin $^{\circledR}$ & $13,1 \pm 2,28 \mathrm{~b}$ & 70,0 \\
Planta Clean $^{\circledR}$ & $16,1 \pm 3,03 \mathrm{~b}$ & 64,3 \\
Mattan Plus $^{\circledR}$ & $36,7 \pm 3,33 \mathrm{c}$ & 49,0 \\
Testemunha & $74,2 \pm 14,02 \mathrm{~d}$ & 0,0 \\
\hline $\mathrm{CV}(\%)$ & 29,65 & \\
\hline DMS & 1,6 & \\
\hline &
\end{tabular}

Além de outros efeitos, os compostos presentes no óleo de nim interferem na alimentação dos insetos, apresentando atividade fagoinibitória e repelente, portanto, a mortalidade dos adultos pode ter ocorrido devido à descontinuidade alimentar que indiretamente provoca alteração no desenvolvimento do inseto (MORDUE; BLACKWELL, 1993; SIMÕES et al., 2007). Portanto, estes efeitos poderiam ser a razão para a mortalidade dos adultos neste experimento com o produto Pironin ${ }^{\circledR}$.

Observou-se que a oviposição foi menor nas folhas nas quais se aplicou Calda fertilizante foliar ${ }^{\circledR}$ (3,3 ovos por folha), diferindo estatisticamente dos demais produtos. Em seguida, a menor oviposição foi observada em folhas tratadas com Pironin ${ }^{\circledR} \mathrm{e}$ Planta clean ${ }^{\circledR}(13,1$ e 16,1 ovos, respectivamente), iguais entre si, mas diferindo do Mattan plus ${ }^{\circledR}$ que, entre os produtos testados, foi o que propiciou a maior oviposição (36,7 ovos), porém diferiu significativamente da testemunha (Tabela 3). Estes resultados sugerem que, no caso dos produtos Pironin $^{\circledR}$ e Calda fertilizante foliar ${ }^{\circledR}$, a menor oviposição pode ter sido causada pela mortalidade das fêmeas antes de ovipositarem (Tabela 2) ou pela presença, nesses produtos, de compostos voláteis, repelentes aos adultos de $B$. tuberculata. Porém, os produtos Planta Clean ${ }^{\circledR}$ e Mattan plus ${ }^{\circledR}$ causaram deterrência a oviposição visto que a redução da oviposição foi proporcionalmente maior que a redução na sobrevivência dos adultos (Tabelas 2 e $3)$.

O óleo de nim, componente do produto Pironin ${ }^{\circledR}$, pode repelir adultos de $B$. tabaci e $B$. tabaci biótipo $\mathrm{B}$ em folhas de algodão tratadas com extratos aquosos de sementes de nim (COUDRIET; PRABHAKER; MEYERDIRK, 1985), folhas de meloeiro tratadas com óleo de nim (AZEVEDO et al., 2005; SILVA; BLEICHER; ARAÚJO, 2003) e em folhas de tomate tratadas com extrato de sementes e folhas de $A$. indica (BALDIN et al., 2007), resultando, em todos os casos, em menor oviposição. Também em tomate, a aplicação de produto comercial à base de nim sobre o solo e sementes, diminuiu significativamente a oviposição de $B$. tabaci pela repelência de adultos do inseto (KUMAR 2008).

Além destas hipóteses, a redução significativa na oviposição de moscas-brancas em folhas tratadas com extratos de nim pode ser devido aos compostos lipídicos presentes no extrato que afetam a oviposição, prejudicando a aderência dos ovos às folhas (QUINTELA; PINHEIRO, 2009). Já o extrato pirolenhoso, também componente do produto Pironin ${ }^{\circledR}$ ativa substâncias do metabolismo secundário das plantas, induzindo a resistência ao ataque dos insetos (TSUZUKI; MORIMTSU; MATSUI, 2000). Da mesma forma, por apresentar 
também ação repelente contra os insetos adultos (AZEVEDO et al., 2005), o produto Pironin ${ }^{\circledR}$ provavelmente impediu ou reduziu a oviposição das fêmeas nas folhas da mandioca, diminuindo assim, o número de ovos encontrados nas folhas.

Informações sobre ação de deterrência em indivíduos da família Aleyrodidae pela Calda fertilizante foliar $^{\circledR}$ são inexistentes. Porém, a aplicação de Calda sulfocálcica, mesma composição da Calda fertilizante foliar $^{\circledR}$, sobre plantas de pimenta, teve ação de mortalidade e repelente sobre adultos do ácaro-branco (Polyphagotarsonemus latus) (VENZON et al., 2006). Segundo Polito (2000), a reação dos compostos da calda aplicada sobre a planta com a água e o gás carbônico, resulta em gás sulfídrico e enxofre coloidal, que confere às caldas sulfocálcicas propriedades acaricidas $\mathrm{e}$ inseticidas.

Os resultados apresentados mostram que alguns PFA, como Calda fertilizante foliar ${ }^{\circledR}$, Planta Clean ${ }^{\circledR} \mathrm{e}$ Pironin ${ }^{\circledR}$ promovem efeito deterrente na oviposição e afetam a sobrevivência de ninfas e adultos da mosca-branca, B. tuberculata em mandioca.

Alguns dos produtos testados apresentaram perspectivas para serem utilizados no controle desse inseto, contudo, testes comparativos das formulações comerciais com os extratos dos vegetais presentes nos componentes destes produtos são importantes e os trabalhos de campo são fundamentais, pois a ação dos produtos pode ser alterada por condições ambientais.

Apesar dos resultados obtidos com os PFA avaliados, e considerando que seu uso justificase por um menor desequilíbrio ambiental e menor resíduo, devem ser também avaliados os efeitos letais e subletais desses produtos sobre os inimigos naturais de ocorrência freqüente no agroecossistema da mandioca.

\section{Agradecimentos}

À Secretaria de Estado de Ciência Tecnologia e Ensino Superior (SETI), pelo apoio financeiro. Ao CNPq e CAPES, pela concessão de bolsa de produtividade e bolsas de mestrado. À Dra. Maria del Pilar Hernandez, do Centro Internacional de Agricultura Tropical (CIAT), Palmira/Colômbia pela identificação da espécie de mosca-branca utilizada no experimento.

\section{Referências}

AGROFIT. Sistema de agrotóxicos fitossanitários. Brasilia, DF: Ministério da Agricultura, Pecuária e Abastecimento. Disponível em: <http:/extranet. agricultura.gov.br/agrofit_cons/principal_agrofit_cons $>$. Acesso em: 15 jun. 2011.

ALVES, S. B.; HADDAD, M. L.; MORAES, R. C. B.; REYES, A. E. L. Correção de mortalidade. 2005. Disponível em: <http://www.lef.esalq.usp.br/cm/intro. php>. Acesso em: 14 jun. 2009.

AZEVEDO, F. R.; GUIMARÃES, J. A.; BRAGA SOBRINHO, R.; LIMA, M. A. A. Eficiência de produtos naturais para o controle de Bemisia tabaci biótipo B (Hemiptera: Aleyrodidae) em meloeiro. Arquivos do Instituto Biológico, São Paulo, v. 72, n. 1, p. 73-79, 2005.

BALDIN, E. L. L.; SOUZA, D. R.; SOUZA, E. S.; BENEDUZZI, R. A. Controle de mosca-branca com extratos vegetais, em tomateiro cultivado em casa-devegetação. Horticultura Brasileira, Brasília, v. 25, n. 4, p. 602-606, 2007.

BELLON, P. P.; PIETROWSKI, V.; ALVES, L. F. A.; RHEINHEIMER, A. R. Técnica para o desenvolvimento de bioensaios com Vatiga manihotae (Drake) (Hemiptera: Tingidae) em laboratório. Arquivos do Instituto Biológico, São Paulo, v. 78, n. 1, p. 115-117, 2011.

BELLOTTI, A. C.; ARIAS, B. V.; VARGAS, O. H.; REYES, J. A. Q.; GUERRERO, J. M. Insectos y acaros dañinos a la yuca y su control. In: OSPINA, B.; CEBALlOS, H. (Ed.). La yuca en el tercer milenio: sistemas modernos de producción, procesamiento, utilizacion y comercialización. Cali: CIAT/CLAYUCA, 2002. p. 160-203.

BELlOTTI, A. C.; SMITH, L.; LAPOINTE, S. L. Recent advances in cassava pest management. Anais $d a$ Sociedade Entomológica do Brasil, Londrina, v. 44, n. 1, p. 343-370, 1999. 
BLEICHER, E.; GONÇALVES, M. E. de C.; SILVA, L. D. da. Efeito de derivados de nim aplicados por pulverização sobre a mosca-branca em meloeiro. Horticultura Brasileira, Brasília, v. 25, n. 1, p. 110-113, 2007.

BUTTERWORTH, J. H.; MORGAN, E. D. Investigation of the locust feeding inhibition of the seeds of the nim tree, Azadirachta indica. Journal of Insect Physiology, Cambridge, v. 17, n. 6, p. 969-977, 1971.

COUDRIET, D. L.; PRABHAKER, N.; MEYERDIRK, D. E. Sweet potato whitefly (Homoptera: Aleyrodidae): effects of neem-seed extract on oviposition and immature stages. Environmental Entomology, Lanhan, v. 14, n. 6, p. 776-779, 1985.

DREYER, M. Nim - a promising natural insecticide for small scale vegetable producers in the Dominican Republic. Dominican Republic: GTZ, 1990. 11 p.

FERREIRA, D. F. SISVAR Sistema para análise de variância para dados balanceados. Lavras: UFLA, $1992.79 \mathrm{p}$.

FOSTER, S. P.; HARRIS, M. O. Behavioral manipulation methods for insect pest-management. Annual Review of Entomology, Palo Alto, v. 42, n. 1, p. 123-146, 1997.

JACOBSON, M. Botanical pesticides: past, present and future. In: ARNASON, J. T.; PHILOGENE, B. J. R.; MORAND, P. (Ed.). Insecticides of plant origin. Washington: American Chemical Society, 1989. p. 110119.

KUMAR, P. Studies on loss of bio-efficacy of two indirect nim application over time (seed and soil) against Bemisia tabaci (Homoptera: Aleyrodidae) under semifield conditions. Journal of Asia-Pacific Entomology, v. 11, n. 4, p. 185-190, 2008.

MARTINEZ, S. S.; LIMA, J.; BOIÇA JUNIOR, A. L. Avaliação agronômica e fitoquímica do nim, Azadirachta indica, de diferentes procedências em vários locais das regiões Sul e Sudeste do Brasil. In: CONGRESSO BRASILEIRO DE ENTOMOLOGIA, 17., 1998, Rio de Janeiro. Anais... Rio de Janeiro: Sociedade Entomológica do Brasil, 1998. p. 831.

MARTINEZ, S. S. O Nim: Azadirachta indica, natureza, usos múltiplos, produção. Londrina: IAPAR, 2002. 142 p.

MORANDI FILHO, W. J.; BOTTON, M.; GRÜTZMACHER, A. D.; GIOLO, F. P.; MANZONI, C. G. Ação de produtos naturais sobre a sobrevivência de Argyrotaenia sphaleropa (Meyrick) (Lepidoptera: Tortricidae) e seletividade de inseticidas utilizados na produção orgânica de videira sobre Trichogramma pretiosum Riley (Hymenoptera: Trichogrammatidae). Ciência Rural, Santa Maria, v. 36, n. 4, p. 1072-1078, 2006.

MORDUE, L. A. J.; BLACKWELL, A. Azaradirachtin: an update. Journal of Insect Physiology, Oxford, v. 39, n. 11, p. 903-924, 1993.

MOREIRA, M. A. B.; FARIAS, A. R.; AlvES, M. C. S.; CARVALHO, H. W. de. Alternativas para o controle da mosca-branca, Aleurothrixus aepim na cultura da mandioca em Sergipe. Aracaju: EMBRAPA, 2006. 4 p. (Comunicado técnico, 56).

OBENG-OFORI, D. Plant oils as grain protectants against infestations of Cryptolestes pusillus and Rhyzopertha dominica in stored grain. Entomologia Experimentalis et Applicata, Dordrecht, v. 77, n. 2, p. 133-139, 1995.

OLIVEIRA, M. R. V.; MORETZSHON, M. C.; QUEIROZ, P. R.; LAGO, W. N. M.; LIMA, L. H. C. Levantamento de moscas-brancas na cultura da mandioca no Brasil. Brasília: Embrapa Recursos Genéticos e Biotecnologia, 2001. 20 p. (Boletim de Pesquisa e Desenvolvimento).

POLITO, W. L. Calda sulfocálcica, bordalesa e viçosa: os fitoprotetores no contexto da trofobiose. Agroecologia Hoje, Botucatu, v. 1, n. 3, p. 20-21, 2000.

QUINTELA, E. D.; PINHEIRO, P. V. Redução da oviposição de Bemisia tabaci biótipo B (Hemiptera: Aleyrodidae) em folhas de feijoeiro tratadas com extratos botânicos. Bioassay, Piracicaba, v. 8, n. 4, p. 1-10, 2009.

RHEINHEIMER, A. R.; BELLON, P. P.; HACHMANN, T.; MIRANDA, A. M.; SCHERER, W.A., PIETROWSKI, V.; ALVES, L. F.; PINTO JUNIOR, A. S. Biologia da mosca-branca (Bemisia tuberculata Bondar) (Hemiptera: Aleyrodidae) em mandioca. Revista Raizes e Amidos Tropicais, Botucatu, v. 5, n. 1, p. 265-269, 2009.

ROJATKAR, S. R.; BHAT, V. S.; KULKARNI, N. M.; JOSHI, V. S.; NAGASAMPAGI, B. A. Tetranortriterpenoids from Azadirachta indica. Phytochemistry, Inglaterra, v. 28, n. 1, p. 203-205, 1989.

SILVA, L. D. da; BLEICHER, E; ARAÚJO, A. C. Eficiência de azadiractina no controle de mosca-branca em meloeiro sob condições de casa de vegetação e campo. Horticultura Brasileira, Brasília, v. 21, n. 2, p. 198-201, 2003.

SCHMUTTERER, H. Properties and potential of natural pesticide from the nim tree, Azadirachta indica. Annual Review of Entomology, Palo Alto, v. 35, n. 1, p. 271-297, 1990. 
SIMÕES, C. M. O.; SCHENKEL, E. P.; GOSMANN, G.; MELLO, J. C. P.; MENTZ, L. A.; PETROVICK, P. R. Farmacognosia, da planta ao medicamento. Porto Alegre: UFRGS, 2007. 1104 p.

TAMM, L.; HÄSELI, A.; FUCHS, J. G.; WEIBEL, F. P.; WYSS, E. Organic fruit production in humid climates of Europe: bottlenecks and new approaches in disease and pest control. Acta-Horticulturae, Leuven, v. 638, n. 1, p. 333-339, 2004.
TSUZUKI, E.; MORIMTSU, T.; MATSUI, T. Effect of chemical compounds in pyroligneus acid on root growth in rice plants. Japonese Journal Crop Science, Bankyoku, v. 66, n. 4, p. 15-16, 2000.

VENZON, M.; ROSADO, M. da C.; PINTO, C. M. F.; DUARTE, V. da S.; EUZÉBIO, D. E.; PALLINI, A. Potencial de defensivos alternativos para o controle do ácaro-branco em pimenta "Malagueta". Horticultura Brasileira, Brasília, v. 24, n. 2, p. 224-227, 2006. 